\title{
High Prevalence of High Risk HPV with Low Frequency of Tp53 Mutations in Esophageal Squamous Cell Carcinoma and Adenocarcinoma Patients in Tangshan China
}

\author{
Vania H. M. Teofilo ${ }^{1}$, Jintao Li ${ }^{1,2}$, Mohammadreza Mohammadzad Mephryar ${ }^{1}$, Si Ye ${ }^{1}$, \\ Shaomei Zhou ${ }^{1}$, Rugang Zhong ${ }^{1}$, Yi Zeng ${ }^{1,2}$ \\ ${ }^{1}$ Beijing Key Lab. of Environmental \& Viral Oncology, College of Life Science \& Bioengineering, Beijing Univ. of Technology, Beijing, China \\ ${ }^{2}$ State Key Laboratory for Infectious Disease Prevention and Control, National Institute for Viral Disease Control and Prevention, Chinese \\ Center for Disease Control and Prevention, Beijing, China
}

Email address:

1jt2000593@163.com (Jintao Li),vtg@emails.bjut.edu.cn (V. H. M. Teofilo)

\section{To cite this article:}

Vania H. M. Teofilo, Jintao Li, Mohammadreza Mohammadzad Mephryar, Si Ye, Shaomei Zhou, Rugang Zhong, Yi Zeng. High Prevalence of High Risk HPV with Low Frequency of Tp53 Mutations in Esophageal Squamous Cell Carcinoma and Adenocarcinoma Patients in Tangshan China. International Journal of Genetics and Genomics. Vol. 4, No. 2, 2016, pp. 11-15. doi: 10.11648/j.ijgg.20160402.12

Received: April 7, 2016; Accepted: May 3, 2016; Published: May 5, 2016

\begin{abstract}
Esophageal cancer, including Squamous Cell Carcinoma and Adenocarcinoma, is one of the most common causes of cancer and cancer death globally, with China alone accounting for about half of the new cases worldwide in 2012. In this study we analyzed the presence of human papilloma virus (HPV) DNA and Tp53 mutations in 52 cancer patients, including 26 Squamous Cell Carcinoma cases, 21 Adenocarcinomas and 5 of non-described histology from Tangshan China. Overall 44 patients were positive for HPV L1 consensus, 28 were positive for HPV16 and 34 for HPV18 $(84.62 \%, 53.82 \%$ and $65.38 \%$ respectively); 23 samples (44.23\%) were positive for both HPV16 and HPV 18. We detected however a very low rate of Tp53 mutations in exons 5 through 8 , which may possible be related to the elevated percentage of high risk HPV. Our findings corroborate our previous study on Esophageal Squamous Cell carcinoma in the same area but a further analysis on other Tp53 exons polymorphisms are necessary to understand the reason behind the low level of Tp53 mutations we reported.
\end{abstract}

Keywords: Esophageal Cancer, HPV, Tp53 Mutations

\section{Introduction}

Esophageal cancer (EC), including Squamous Cell Carcinoma (ESCC) and Adenocarcinoma (AC), is the eighth most common cancer worldwide, and the sixth most common cause of cancer death [1]. While the prognosis remains poor, the incidence rate has increased [2] with an estimated 456,000 new cases and 400,000 deaths in 2012 [1].

The incidence rate varies greatly worldwide with about $80 \%$ of new cases occurring in less developed countries, mainly Southern Africa and the "esophageal cancer belt" area which extends from Eastern Central Asia to the Middle East [3-5]. Of these areas, China alone accounted for about half of the new ESSC cases worldwide in 2012 [3], with an incidence rate of $22.4 / 100000$ and a mortality rate of $16.77 / 100000$ [6]. The etiology of both histological types is thought to be multifactorial with a number of etiological agents such as, environmental and dietary factors, genetic alterations and infection agents believed to be implicated. One of the genetic alterations associated with EC carcinogenesis is the disruption of the tumor suppressor gene Tp53. The dysfunction of Tp53 is a hallmark of human cancers and is mainly caused by somatic missense mutations, that occur primarily in the DNA biding domain $[8,9]$, leading to the accumulation of mutant $\mathrm{Tp} 53$ protein. These mutations have been shown to occur in at least $50 \%$ of esophageal cancer cases and have been detected in both ESCC and EAC [10-13].

Tp53 regulatory function can also be suppressed by the activities of Human papilloma virus (HPV) E6 onco-protein which induces p53 degradation by forming a trimeric complex with p53 and the cellular E3 ubiquitin ligase E6-associated protein (E6AP), that leads to the ubiquitination and proteasomal 
degradation of Tp53 $[14,15]$. Although the association between HPV and ESCC has been observed since 1982 by Syrjanen [16], the role of HPV in the carcinogenesis of EC remains unclear. However, in a 2013 meta-analysis study, comparing the results of 21 case-control studies, concluded that HPV DNA was present in $35 \%$ of the ESCC samples and that HPV increases the risk of HPV three-fold [17].

Previous studies in China have demonstrated a high prevalence of Tp53 mutations, while results on HPV prevalence have been contradictory, with some scoring very high prevalence and others concluding a small role for HPV in EC carcinogenesis [18-21]. Additionally, these studies have focused mostly on ESCC, and HPV prevalence on Chinese AC patients has been poorly addressed.

In this study we aim to evaluate the prevalence of HPV DNA and Tp53 point mutations in both ESCC and AC patients from Tangshan, China, an area previously reported by our group as having a high prevalence of high risk HPV types 16 and 18 .

\section{Materials and Methods}

\subsection{Tumor Samples and DNA Extraction}

FFPE tissue blocks from esophageal cancer patients specimens were obtained from a pathology department in Tangshan, China from 2007 to 2011. In total 52 samples were used in our study: 26 ESCC, $21 \mathrm{AC}$ and 5 of non-described histology. Histological types, gender and age of patients were also provided by the department.

DNA extraction from FFPE the tissue blocks was performed as per our usual laboratory procedure previously described in Mehryar et al [22].

\subsection{HPV Detection}

To evaluate the performance of the extraction, DNA was amplified for the $\beta$-globin gene using $\mathrm{PC} 04 / \mathrm{GH} 20$ primer set [(5'- CAA CTT CAT CCA CGT TCA CC -3'/ 5'- GAA GAG CCA AGG ACA GGT AC- 3) $62^{\circ} \mathrm{C}$ annealing temperature]. Subsequently, positive samples for the $\beta$-globin, were screened for L1 consensus with GP5+/GP6+ primer set [(5'- TTT GTT ACT GTG GTA GAT ACT AC3'/ 5' - CTT ATA CTA AAT GTC AAA TAA AAA G- 3'), $55^{\circ} \mathrm{C}$ annealing temperature] to detect a wide range of $\mathrm{HPV}$ types [23]. For the amplification of HPV types 16 and 18 type-specific primers were used: HPV16E6F/R [(5'- CAA CAA GAC ATA CAT CGA CC -3'/ 5'- CAA CAA GAC ATA CAT CGA CC $\left.-3^{\prime}\right), 60^{\circ} \mathrm{C}$ annealing temperature] and HPV18E6F/R [(5'- CAC TTC ACT GCA AGA CAT AGA-3'/5'- GTT GTG AAA TCG TCG TTT TTC A -3'), $55^{\circ} \mathrm{C}$ annealing temperature]. Takara ExTaq ${ }^{\circledR}$ Hot Start PCR kit (Takara Inc., Otsu, Shiga, Japan) was used for all the primer sets. All PCR procedures were repeated twice, and first PCR product was used as template for the second to guarantee that samples with low viral DNA load would be identified.

\subsection{Detection of Tp53 Mutations}

PCR amplification of exons 5 through 8, was performed according to the 2010 update of the IARC protocol. Primers and PCR conditions are described in detail and are available on the TP53 IARC database website: http://p53.iarc.fr /Download/TP53_DirectSequencing_IARC.pdf. The PCR mix was made, however, with Takara Ex Taq ${ }^{\circledR}(\mathrm{Mg} 2+$ free buffer) kit for exons, 5, 6, and 8 and Takara Ex Taq ${ }^{\circledR}$ Hot Start PCR kit for exon 7. Samples were analyzed by bidirectional sequencing and results were compared by DNAMAN software with the reference sequence NC_000017-9 from http://p53.iarc.fr/TP53Sequence_NC_00 0017-9.aspx. Codon order and amino acid sequence were also compared with the sequence provided by the IARC database [24].

\subsection{DNA Sequencing}

HPV and Tp53 PCR-positive products were sequenced and analyzed using the T7 sequence version 2.0 DNA PCR product sequencing kit (Affymetrix, Santa Clara, CA, United States) to identify the HPV types and any sequence variations. The nucleotide sequences were subsequently confirmed using the Basic Local Alignment Search Tool (BLAST).

\section{Results}

\subsection{Demographics}

For demographic analysis, only samples with known histological origin were used totaling 47 patients (26 ESCC and $21 \mathrm{AC})$. The patients were separated per each histological type according to gender and age. A total of 38 $(81 \%)$ patients were men and only $9(19 \%)$ were women. The same pattern is observed when analyzing both histological types separately with ESCC scoring $21(80.8 \%)$ men vs. 5 (19.2\%) women and AC patients scoring 17 (81\%) men vs. 4 (19\%) women.

The patients age varied from 40 to 79 years and the majority of the patients were older than $60 \mathrm{yr}$. [32 (68.1\%)], with a total average age of $61.6 \mathrm{yr}$. When considering gender and histological types in separate groups, ESCC male patients had a younger average age (58.7 yr.) and had 50/50 distribution for younger and equal/older than $60 \mathrm{yr}$. groups (Table 1).

Table 1. Age distribution per subtype.

\begin{tabular}{llll|ll}
\hline \multicolumn{6}{l}{ Age Distribution (\%) and Average Age (yr.) } \\
\hline & $<\mathbf{6 0}$ yr. & $\mathbf{2 6 0}$ yr. & Men & Women & Overall \\
\hline ESCC & $13(50 \%)$ & $13(50 \%)$ & 58.7 & 62.8 & 59.5 \\
AC & $2(9.5 \%)$ & $19(90.5 \%)$ & 64.2 & 64.5 & 64.2 \\
Total & $15(31.9 \%)$ & $32(68.1 \%)$ & 61.1 & 63.6 & 61.6 \\
\hline
\end{tabular}

\subsection{Detection of HPVDNA}

G5+/G6+ and HPV type specific primer set were used in all 52 EC samples, including 5 of undetermined histological type and the results are summarized in Figure 1. The 
prevalence of HPV L1 sequence for all 52 samples was $84.62 \%(44 / 52)$ and the frequency of HPV 16 and 18 type-specific sequences was $53.82 \%(28 / 52)$ and $65.38 \%$ (34/52) respectively. Twenty three samples (44.23\%) tested positive for both HPV 16 and 18 .

L1 sequence was present in $88.46 \%(23 / 26)$ of ESCC specimens and frequency of HPV16 and 18 was $53.85 \%$ $(14 / 26)$ and $73.08 \%(19 / 26)$. Twelve samples (46.15\%) were positive for both HPV16 and HPV18 (Figure 2).

In the AC group 76.12\% (16/21) of the samples had positive results for the G5+/G6+ primer set, while $57.14 \%$ (12/21) tested positive for HPV16, 66.67\% (14/21) were positive for HPV18 and 52,38\% (11/21) were double positives (Figure 3).

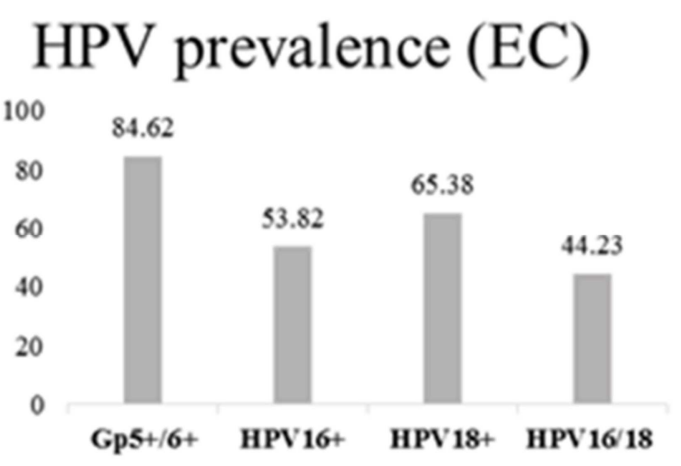

Figure 1. Percentage of HPV viral DNA prevalence in all samples.

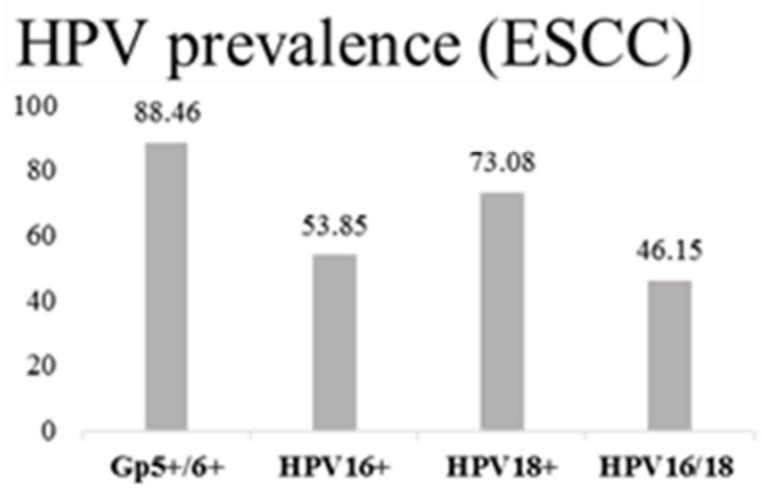

Figure 2. Percentage of HPV viral DNA prevalence in ESCC subtype.

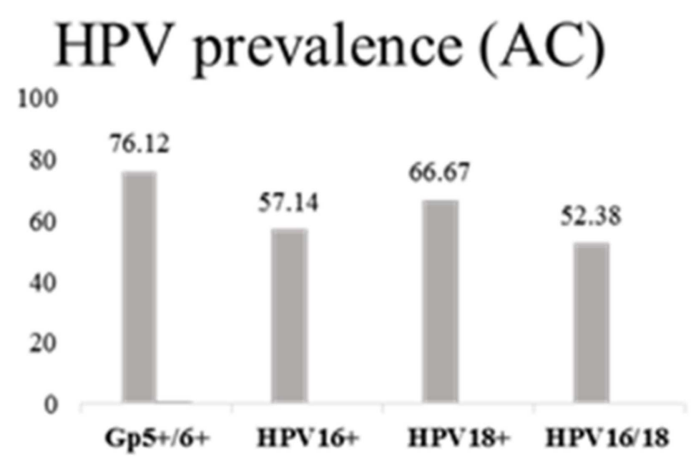

Figure 3. Percentage of HPV viral DNA prevalence in AC subtype.

\subsection{Detection of TP53 Mutations}

The number of Tp53 mutations was extremely low with only 2 mutations detected for 52 samples (3.85\%): Patient \#2010-5895 had a missense mutation on Exon 7, codon 245 $($ GGC $\rightarrow$ TGC / Gly $\rightarrow$ Cys) and patient \#117240T had a nonsense mutation on Exon 8 codon 287 (GAG $\rightarrow$ TAG / Glu $\rightarrow$ Stop).

\section{Discussion}

China has one of the world's largest numbers of esophageal cancer cases and many studies have been carried out to understand the factors behind the high incidence rate and the role of HPV infection and mutations in the Tp53 gene. The presence of HPV DNA in ESCC has been highly controversial with studies reporting very different levels of HPV prevalence. [18-21]. Even samples from the same area or same laboratory may report conflicting results [21]. Detection methodology differences may be at bottom of this discrepancy, and PCR based detection is advised since it's considered a more sensitive method.

In addition, Guo et al. noted that HPV copy number in esophageal cancer specimens was at least 2 orders of magnitude lower than that in cervical cancer and, in order to avoid, false low levels of detection, L1 PCR should be performed twice [21].

Following this suggestion we observed on our lab a high prevalence of HPV DNA, in this study. These results are consistent with a 2015 meta-analysis of Chinese case-control studies that highlighted more than a six fold increased risk of esophageal cancer in the presence of HPV 16 infection [25].

These studies have, however, been primarily focused on ESCC, since it's the most common histological type of EC and HPV 16. In this study we analyzed samples on both ESCC and AC origin to determine the prevalence high risk HPV16 and 18.

In our samples, we observed a higher prevalence of HPV 18 DNA than HPV16 in both ESCC and AC (73.08\% vs. $53.85 \%$ and $66.67 \%$ vs. $57.14 \%$, respectively) and many samples presented both types of high risk HPV (46.15\% in ESCC, $52.38 \%$ in AC and $44.23 \%$ in total). These results suggest that both high risk HPVs may be involved in ESCC and AC carcinogenesis in Tangshan, China.

On the other hand, we detected a very low prevalence of TP53 mutations which is contrary to previously reported data for high incidence areas [26, 27]. To our knowledge only previous studies in South Africa and Kenya reported a low prevalence of $\mathrm{Tp} 53$ mutations in a high incidence area [28, 29] and, even these studies reported a prevalence rate of $17-25 \%$ and $39.9 \%$.

These results are unexpected since Tp53 mutations are considered a hallmark of esophageal cancer and, contrary to cervical carcinoma in which Tp53 mutations gene are rare in cases associated with HPV infections, these events are not mutually exclusive in esophageal carcinoma [30]. Some studies did report an inverse relationship between Tp53 mutations and HPV infections in ESCC carcinoma [31] which might explain our low mutation rate in the presence of a high prevalence of high risk HPVs. 
It is important to note that, our study was limited to exons 5 to 8 , since they contain about $90 \%$ of known dysfunctional Tp53 mutations, and we cannot exclude the possibility of mutational events in the other exons of the gene, Other limitations we encountered included the lack of detailed information regarding other risk factors such as alcohol and tobacco consumption, dietary habits and economical background that are implicated in esophageal cancer carcinogenesis.

In summary, our results show, that the role of HPV infection in the development of esophageal carcinomas of different histological type, cannot be disregarded and so, preventive measures such as HPV screening and improving vaccination policies using either the quadrivalent Gardasil vaccine that targets HPV6, 11,16 and 18 or the bivalent Cervarix vaccine that targets HPV16 and 18, could be beneficial in addressing the high incidence of esophageal carcinoma.

\section{Conclusion}

The results presented in this study corroborate our previous observations of an elevated presence of HPV viral DNA in Tangshan patients and are also in accordance with other studies that have indicated that high risk HPVs may play a role in esophageal cancer in China. It is then advisable to consider HPV preventions measurements as possible elements in addressing the high rates of the malignancy in China.

Our data did not concur however with previous studies on Tp53 mutation in the country. This might be due to a high level of HPV infection however we cannot rule out the limitations in our own study. In the future a further analysis of Tp53 mutation in different exons and polymorphisms in the gene may provide a better clue as to the low prevalence we have reported here.

\section{Acknowledgements}

This study was supported by the following grants:

Grant sponsor: Natural science foundation of Beijing. Grant number: 5162003.

Grant sponsor: Beijing University of Technology Foundation, Grant number: 015000514314004.

\section{References}

[1] J. Ferlay, I. Soerjomataram, M. Ervik, R. Dikshit, S. Eser, C. Mathers, M. Rebelo, D. M. Parkin, D. Forman and F. Bray, GLOBOCAN 2012 v1.0, Cancer Incidence and Mortality Worldwide: IARC Cancer Base No. 11 [http://globocan.iarc.fr/Pages/fact_sheets_cancer.aspx]. Lyon, France: International Agency for Research on Cancer; 2013. Available from: http://globocan.iarc.fr, accessed on $11 / 03 / 2016$.

[2] G. Edgren, H. O. Adami, E. Weiderpass and O. Nyren, "A global assessment of the oesophageal adenocarcinoma epidemic," Gut, 2013, vol. 62(10), pp.1406-14.
[3] M. Arnold, I. Soerjomataram, J. Ferlay and D. Forman, "Global incidence of oesophageal cancer by histo-logical subtype in 2012," Gut, 2015, vol. 64, pp. 381-387.

[4] M. P. Curado, B. Edwards, H. R. Shin, H. Storm, J. Ferlay, M. Heanue and P. Boyle, Cancer incidence in five continents, vol. IX, IARC Scientific Publications No. 160. Lyon: IARC, 2007.

[5] Y. Zhang, "Epidemiology of esophageal cancer," World J. Gastroentrol, 2013, vol. 19(34), pp. 5598-5606.

[6] W. Chen, R. Zheng, S. Zhang, P. Zhao, H. Zeng, X. Zou, and J. He, “Annual report on status of cancer in China, 2010,” Chin. J. Cancer Res., 2014, vol. 26(1), pp. 48-58.

[7] K. J. Napier, M. Scheerer, and S. Misra, "Esophageal cancer: A review of epidemiology, pathogenesis, staging workup and treatment modalities," World J. Gastrointest. Oncol., 2014, vol. 6(5), pp. 112-120.

[8] M. Olivier, M. Hollstein, and P. Hainaut, "TP53 mutations in human cancers: origins, consequences and clinical use". Cold Spring Harb. Prespect. Biol., 2010, vol. 2 a0001008.

[9] C. Whibley, P. D. Pharoah, and M. Hollstein, "p53 polymorphisms: cancer implications," Nat. Rev. Cancer, 2009, vol. 9, pp. 99-107.

[10] M. C. Hollstein, R. A. Metcalf, J. A. Welsh, R. Montesano, and C. C. Harris, "Frequent mutation of the p53 gene in human esophageal cancer," Proc. Natl. Acad. Sci. USA, 1990, vol. 87(24), pp. 9958-9961.

[11] M. C. Hollstein, L. Peri, A. M. Mandard, J. A. Welsh, R. Montesano, R. A. Metcalf, M. Bak and C. C. Harris, "Genetic analysis of human esophageal tumors from two high incidence geographic areas: frequent $\mathrm{p} 53$ base substitutions and absence of ras mutations," Cancer Res., 1991, vol. 51(15) pp. 4102-4106.

[12] N. Agrawal, Y. Jiao, C. Bettegowda, S. M. Hutfless, Y. Wang, S. David, Y. Cheng, W. S. Twaddell, N. L. Latt, E. J. Shin, L. D. Wang, L. Wang, W. Yang, V. E. Velculescu, B. Vogelstein, N. Papadopoulos, K. W. Kinzler, and S. J. Meltzer, "Comparative genomic analysis of esophageal adenocarcinoma and squamous cell carcinoma," Cancer Discov., 2012, vol. 2(10), pp. 899-905.

[13] A. M. Dulak, P. Stojanov, S. Peng, M. S. Lawrence, C. Fox, C. Stewart, S. Bandla, Y. Imamura, S. E. Schumacher, E. Shefler, A. McKenna, S. L. Carter, K. Cibulskis, A. Sivachenko, G. Saksena, D. Voet, A. H. Ramos, D. Auclair, K. Thompson, C. Sougnez, R. C. Onofrio, C. Guiducci, R. Beroukhim, Z. Zhou, L. Lin, J. Lin, R. Reddy, A. Chang, R. Landrenau, A. Pennathur, S. Ogino, J. D. Luketich, T. R. Golub, S. B. Gabriel, E. S. Lander, D. G. Beer, T. E. Godfrey, G. Getz, and A. J. Bass, "Exome and whole-genome sequencing of esophageal adenocarcinoma identifies recurrent driver events and mutational complexity," Nat. Genet., 2013,vol. 45(5), pp. 478-86.

[14] C. A. Moody and A. L. Laimins, "Human papillomavirus oncoproteins: pathways to transformation," Nat. Rev. Cancer, 2010, vol. 10, pp. 550-560.

[15] H. L. Howie, R. A. Katzenellenbogen and D. A. Galloway, "Papillomavirus E6 proteins. Minireview," Virol., 2001, vol. 384, pp.324-334.

[16] K. J. Syrjanen, "Histological changes identical to those of condylomatous lesions found in esophageal squamous cell carcinomas," Arch Geschwolstforsh, 1982, vol. 15, pp. 415-425. 
[17] S. S. Liyanage, B. Rahman, I. Ridda, A. T. Newall, S. N. Tabrizi, S. M. Garland, E. Segelov, H. Seale, P. J. Crowe, A. Moa, and C. R. Macintyre, "The aetiological role of human papillomavirus in oesophageal squamous cell carcinoma: A meta-Analysis," PLoS One, 2013, vol. 8(7), pp. 1-12.

[18] J. Koshiol, W. Q. Wei, A. R. Kreimer, W. Chen, P. Gravitt, J. S. Ren, C. C. Abnet, J. B. Wang, F. Kamangar, D. M. Lin, M. von Knebel-Doeberitz, Y. Zhang, R. Viscidi, G. Q. Wang, M. L. Gillison, M. J. Roth, Z. W. Dong, E. Kim, P. R. Taylor, Y. L. Qiao, and S. M. Dawsey, "No role for human papillomavirus in esophageal squamous cell carcinoma in China," Int. J. Cancer, 2010, vol. 127(1), pp. 93-100.

[19] F. Kamangar, Y. L. Qiao, J. T. Schiller, S. M. Dawsey, T. Fears, X. D. Sun, C. C. Abnet, P. Zhao, P. R. Taylor, and S. D. Mark, "Human papillomavirus serology and the risk of esophageal and gastric cancers: results from a cohort in a high-risk region in China,” Int. J. Cancer, 2006,vol. 119(3), pp. 579-584.

[20] D. H. Zhang, Q. Y. Zhang, C. Q. Hong, J. Y. Chen, Z. Y. Shen, and $\mathrm{Y}$. Zhu, "Prevalence and association of human papillomavirus 16, Epstein-Barr virus, herpes simplex virus-1 and cytomegalovirus infection with human esophageal carcinoma: a case-control study," Oncol. Rep., 2011, vol. 25(6), pp. 1731-1738.

[21] F. Guo, Y. Liu, X. Wang, Z. He, N. S. Weiss, M. M. Madeleine, F. Liu, X. Tian, Y. Song, Y. Pan, T. Ning, H. Yang, X. Shi, C. Lu, H. Cai, and Y. Ke,"Human papillomavirus infection and esophageal squamous cell carcinoma: a case-control study," Cancer Epidemiol. Biomarkers Prev., 2012, vol. 21(5), pp.780-785.

[22] M. M. Mehryar, S. Y. Li, H. W. Liu, F. Li, F. Zhang, Y. B. Zhou, Y. Zeng, and J.T. Li, "Prevalence of human papillomavirus in esophageal carcinoma in Tangshan, China," World J. Gastroenterol., 2015, vol. 21(10), pp. 2905-2911.

[23] W. Qu, G. Jiang, Y. Cruz, C. J. Chang, G. Y. Ho, R. S. Klein and R. D. Burk, "PCR detection of human papillomavirus: comparison between MY09/MY11 and GP5+/GP6+ primer systems, ”J. Clin. Microbiol., 1997, vol. 35, pp. 1304-1310.

[24] A. Petitjean, E. Mathe, S. Kato, C. Ishioka, S. V. Tavtigian, P. Hainaut and M. Olivier, "Impact of mutant P53 functional properties on Tp53 Mutation Patterns and Tumor Phenotype: Lessons from recent developments in the Iarc Tp53 database,"
Hum.Mutat., 2007, vol. 28, pp. 622-629. (R17, November 2013 version).

[25] S. K. Zhang, L. W. Guo, Q. Chen, M. Zhang, S. Z. Liu, P. L. Quan, J. B. Lu, and X. B. Sun, "The Association between Human Papillomavirus 16 and Esophageal Cancer in Chinese Population: A Meta-Analysis," BMC Cancer,2015,vol. 15, pp. 1096.

[26] B. Abedi-Ardekani,, F. Kamangar, M. Sotoudeh, S. Villar, F. Islami, K. Aghcheli, D. Nasrollahzadeh, N. Taghavi, S. M. Dawsey, C. C. Abnet, S. M. Hewitt, S. Fahimi, F. Saidi, P. Brennan, P. Boffetta, R. Malekzadeh, and P. Hainaut, "Extremely high Tp53 mutation load in esophageal squamous cell carcinoma in Golestan Province, Iran." PLoS One, 2011, vol. 6(12), e29488.

[27] W. Cao, X. Chen, H. Dai, H. Wang, B. Shen, D. Chu, T. McAfee, and Z. F. Zhang. "Mutational spectra of P53 in geographically localized esophageal squamous cell carcinoma groups in China," Cancer, 2004, vol. 101(4), pp. 834-844.

[28] K. Patel, S. Mining, J. Wakhisi, T. Gheit, M. Tommasino, G. Martel-Planche, P. Hainaut, and B. Abedi-Ardekani, "Tp53 mutations, human papilloma virus DNA and inflammation markers in esophageal squamous cell carcinoma from the rift valley, a high-Incidence area in Kenya," BMC Res. Notes, 2011, vol. 4, pp. 469.

[29] W. Gamieldien, T. C. Victor, D. Mugwanya, A. Stepien, W. C. Gelderblom, W. F. Marasas, D. H. Geiger and P. D. van Helden, "p53 and p16/CDKN2 gene mutations in esophageal tumors from a high-incidence area in South Africa" Int. J. Cancer, 1998, vol. 78, pp. 544-549.

[30] F. Chang, S. Syrjanen, A. Tervahauta, K. Kurvinen, L. Wang, and K. Syrjinen, "Frequent mutations of P53 gene in oesophageal squamous cell carcinomas with and without human papiliomavirus (Hpv) involvement suggest the dominant role of environmental carcinogens in oesophageal carcinogenesis," Br. J. Cancer,1994, vol. 70, pp. 346-351.

[31] S. Katiyar, S. Hedau, N. Jain, P. Kar, M. S. Khuroo, J. Mohanta, S. Kumar, V. Gopalkrishna, N. Kumar and B. C. Das, "P53 gene mutation and human papillomavirus (Hpv) infection in esophageal carcinoma from three endemic geographic regions of India." Cancer Lett., 2005, vol. 218, pp. 69-79. 